Original Research Article

\title{
Study of drug utilization and antimicrobial prescription pattern of indoor paediatrics cases in tertiary care hospital
}

\author{
Vivek Virbhan Bamel ${ }^{1 *}$, Savita Ramesh Shahani ${ }^{1}$, Nimain C. Mohanty ${ }^{2}$
}

${ }^{1}$ Department of Pharmacology, ${ }^{2}$ Department of Paediatrics, MGM Medical College, Navi Mumbai, Maharashtra, India

Received: 16 October 2017

Revised: 27 October 2017

Accepted: 24 November 2017

\section{*Correspondence to:}

Dr. Vivek Virbhan Bamel, Email: viveksinghbame190@ gmail.com

Copyright: (C) the author(s), publisher and licensee Medip Academy. This is an openaccess article distributed under the terms of the Creative Commons Attribution NonCommercial License, which permits unrestricted noncommercial use, distribution, and reproduction in any medium, provided the original work is properly cited.

\begin{abstract}
Background: Infectious disease represent a major cause of mortality and morbidity in India. Pattern of use of antibiotics need to be studied separately as they vary from adult, however there is limited data is available, Therefore the aim of our study is to observe the drug utilization and antimicrobial prescription pattern and drug utilization in department of pediatrics at our tertiary care hospital.
\end{abstract}

Methods: This was a prospective observational study carried out among indoor patients admitted to paediatric ward after obtaining approval by Institutional Ethics Committee. Written informed consent obtained from a parent or legal guardian of participants.

Results: 100 patients were enrolled. The maximum number of antibiotics were prescribed from cephalosporin class $(75 ; 43.6 \%)$ out of which ceftriaxone $(42 ; 56 \%)$, Cefixime $(20 ; 26.6 \%)$, cefotaxim $(11 ; 14.6 \%)$, cefpodoxime $(3 ; 4 \%)$, cefazolin $(1 ; 1.33 \%)$. The majority of cephalosporins were prescribed in Gastrointestinal tract infections $(60.66 \%)$ followed by respiratory $(27.2 \%)$ and least in cvs $(2.66 \%)$, secondly higher group of antimicrobial prescribed was from Aminopenicillin class accounting of $(36 ; 21.9 \%)$ which includes amoxiclav $(35 ; 97.2 \%)$, ampicillin $(1 ; 2.7 \%)$ and other beta lactamase $(3 ; 1.7 \%)$ consisting meropenem $(2 ; 66.7 \%)$, piperacillin with tazobatcam $(1 ; 33.3 \%)$. The majority of aminopenicillin were prescribed in respiratory tracts infections and very less in other systems. Miscellaneous drugs used in respiratory conditions were bronchodilator, systemic steroid and nasal decongestant; in CNS disorders antiepileptic and diuretics; in cardiovascular disease NSAID, inotropic, antihypertensive, diuretics; in genitourinary tract infections alkalizing agent and steroid and in GIT disorders minerals, rehydration fluids, antispasmodic probiotic were used.

Conclusions: We conclude that commonly prescribed drugs were from 3rd generation cephalosporins followed from extended spectrum penicillin with $\beta$ lactmase inhibitor. Majority of the antimicrobials are used empirically.

Keywords: Antimicrobial prescription pattern, Drug utilization

\section{INTRODUCTION}

Drug utilization research is defined by the World Health organisation (WHO) in 1977, as "The marketing, distribution, prescription and use of a drug in society, with special emphasis on the resulting medical, social and economic consequences". ${ }^{1}$
Drug utilization research can be divided into:

- Descriptive (Quantitative) and

- Analytical (Qualitative) studies

In the present study we are emphasising over Descriptive (quantitative) studies on finding out pattern of drug 
utilization and to identify problems deserving more detailed studies.

Antibiotics are one of the commonly prescribed drugs in pediatrics. Because of an overall rise in health care cost, lack of uniformity in drug prescribing and the emergence of antibiotic resistance, monitoring and control of antibiotic use are of growing concern and strict antibiotic polices should be warranted. ${ }^{2}$

Pediatric population is prone to suffer from recurrent infections in the various system of the body. Lower respiratory tract infections are the leading cause of death in children below 5 five years of age. ${ }^{3}$ The use of antimicrobial agents, especially antibiotics has become a routine practice for the treatment of pediatric illnesses. Higher incidence of infections in infants and children as compared to adults leads to higher use of antimicrobials and contribute to an overall increase in healthcare costs as well as potentially severe adverse drug reactions. Several professional societies have issued guidelines designed to reduce the use of antibiotics world- wide by means of various control strategies.

Several studies focusing on antibiotic prescribing attitudes in hospitalized children indicate that approximately $35 \%$ of infants and children admitted to hospitals receive antibiotics and widespread misuse has been reported. ${ }^{4}$

Overuse of antibiotics can result in high prevalence of resistance to commonly prescribed antibiotics, necessitating the use of more expensive second- or third line agents. Thus, the judicious use of antibiotic is therefore an important way to reduce the problem of antimicrobial resistance (Use of Antibiotics in Paediatric Care). Rational use of drugs forms the corner stone of successful implementation of rational use of medicine. ${ }^{5}$

\section{WHO drug use indicators}

In 1993, who identified 12 core indicators and 7 complementary indicators, which can be used to describe drug use pattern in a country, region or individual health facility. ${ }^{6}$ Advantage of these indicators are that we can compare the prescribing pattern of different settings to assess the effect of given indicators for improvement of drug use. Authors have used Prescribing indicator, which includes percentage of encounters with antibiotic use.

Thus, the aim for the present study is to observe drug utilization with special emphasize on the antibiotic prescription pattern and analyze it as per WHO prescribing indicator and compare it with national guidelines in pediatric department at MGM hospital.

\section{METHODS}

This study was prospective observational study carried out in indoor patients admitted to paediatric ward of a tertiary care hospital from (July 2016 to March 2017) after obtaining approval from Institutional Ethics Committee. The participants were explained about the purpose of the study and voluntary written informed consent was obtained from legal accepted representatives. Participants were from new born to 18 years were recruited.

\section{Inclusion criteria}

All hospitalized pediatric population and the children whose parents or LAR had given consent.

\section{Exclusion criteria}

- Parents did not give consent

- Immunocompromised patients

- Grade 4 malnourished children

- Malignancies

- Less than 34 weeks were excluded

\section{RESULTS}

During 10 months of study a total of 100 patients were between age group of 6 months to 14 years with median age of 6 years with male to female ratio (54:46) who were admitted to paediatric ward were enrolled. During entire tenure of study total 172 antimicrobials were prescribed, details of which have been shown in (Table 1).

The maximum number of antibiotics were prescribed from cephalosporin class $(75 ; 43.6 \%)$ which were ceftriaxone $(42 ; 56 \%)$, Cefixime $(20 ; 26.6 \%)$, cefotaxim $(11 ; 14.6 \%)$, cefpodoxime $(3 ; 4 \%)$, cefazolin $(1 ; 1.33 \%)$. The majority of cephalosporins were prescribed in Gastrointestinal tract infections $(60.66 \%)$ followed by respiratory $(27.2 \%)$ and least in cvs $(2.66 \%)$, secondly higher group of antimicrobial prescribed was from Aminopenicillin class accounting $(36 ; 21.9 \%)$ which includes amoxiclav $(35 ; 97.2 \%)$, ampicillin $(1 ; 2.7 \%)$ and other beta lactamase $(3 ; 1.7 \%)$ consisting meropenem $(2 ; 66.7 \%)$, piperacillin with tazobatcam $(1 ; 33.3 \%)$. The majority of aminopenicillin were prescribed in respiratory tracts infections and very less in other systems.

Overall the highest antimicrobial was used in respiratory tract infections $(66 ; 38.37 \%)$ followed by Gastrointestinal tract system $(63 ; 36.62 \%)$ followed by central nervous system $(13 ; 7.5 \%)$ similar percentage observed in genitourinary and pyrexia whereas in cardiovascular system $(4 ; 2.3 \%)$ while no antimicrobial were prescribed in musculoskeletal system. Among the total number of antimicrobial prescriptions for Respiratory tract, the highest class was aminopenicillin flowed by cephalosporin, aminiglycoside, antitubercular and macrolides. For CNS highest number prescribed were cephalosporin followed by antitubercular, amioglycoside and other beta lactam. For pyrexia the highest number of prescribed antimicrobial was from cephalosporin followed by aminopenicillin and antimalarial. For Gastrointestinal tract infections cephalosporin, followed by metronidazole, aminoglycoside and antitubercular drugs. 
Table 1: Antimicrobial prescription pattern in Wards according to class.

\begin{tabular}{|c|c|c|c|c|c|c|c|c|}
\hline Antimicrobials & $\mathbf{R S}$ & CNS & CVS & GIT & GUT & MSK & PYREXIA & Total \\
\hline Cephalosporins ${ }^{\#}$ & 18 & 6 & 2 & 38 & 5 & 0 & 6 & $75(43.6 \%)$ \\
\hline Aminopenicillin* & 27 & 0 & 2 & 1 & 2 & 0 & 4 & $36(21.9 \%)$ \\
\hline Other betalactamase & 1 & 1 & 0 & 0 & 1 & 0 & 0 & $3(1.7 \%)$ \\
\hline Aminoglycoside & 6 & 1 & 0 & 5 & 2 & 0 & 0 & $14(8.13 \%)$ \\
\hline Metronidazole & 0 & 1 & 0 & 15 & 0 & 0 & 0 & $16(9.3 \%)$ \\
\hline Flouroquinolone & 0 & 0 & 0 & 0 & 1 & 0 & 0 & $1(.58 \%)$ \\
\hline Antitubercular & 6 & 4 & 0 & 4 & 0 & 0 & 0 & $14(8.13 \%)$ \\
\hline glycopeptide & 1 & 0 & 0 & 0 & 0 & 0 & 0 & $1(.58 \%)$ \\
\hline Macrolides & 4 & 0 & 0 & 0 & 0 & 0 & 0 & $4(2.32 \%)$ \\
\hline Acyclovir & 0 & 0 & 0 & 0 & 0 & 0 & 0 & 0 \\
\hline Oxazolidieones & 2 & 0 & 0 & 0 & 0 & 0 & 0 & $2(1.16 \%)$ \\
\hline Co-trimoxazole & 0 & 0 & 0 & 0 & 0 & 0 & 0 & 0 \\
\hline Amphotrecin B & 0 & 0 & 0 & 0 & 0 & 0 & 0 & 0 \\
\hline Chloramphenicol & 1 & 0 & 0 & 0 & 0 & 0 & 0 & $1(.58 \%)$ \\
\hline Sulfonamide & 0 & 0 & 0 & 0 & 1 & 0 & 0 & $1(.58 \%)$ \\
\hline Tetracycline & 0 & 0 & 0 & 0 & 1 & 0 & 0 & $1(.58 \%)$ \\
\hline Antimalarial & 0 & 0 & 0 & 0 & 0 & 0 & 3 & $3(1.7 \%)$ \\
\hline Total & 66 & 13 & 4 & 63 & 13 & 0 & 13 & 172 \\
\hline
\end{tabular}

\# Cephalosporins included ceftriaxone (42), cefixime (20), cefpodoxime (3), cefotaxime (11), cefazolin (1), ${ }^{*}$ Amonopenicillin includes co-amoxiclav (35), Ampicillin (1), Other betalactamase includes meropenem (2), piperacillin with tazobactam (1) and glycopeptides is vancomycin (1), Aminoglycoside includes Amikacin (10), tobramycin (1), netillimycin (1) Gentamycin (2). RS = respiratory system, $\mathrm{CNS}=$ Central nervous system, $\mathrm{CVS}=$ cardiovascular system, GIT $=$ Gastrointestinal tract infections, GUT =Genitourinary tract infections, MSK $=$ musculoskeleton system, Pyrexia $=$ fever

Table 2: System wise miscellaneous drugs prescription pattern.

\begin{tabular}{|lllll|}
\hline RS & GIT & CVS & CNS & Genitourinary \\
\hline bronchodilator & Minerals & NSAID & Antiepileptic & Alkalizing agent \\
\hline Inhaled steroid & Rehydrating fluids & Inotropes & & steroid \\
\hline & Antispasmodic & Diuretic & & \\
\hline & Probiotic & & \\
\hline
\end{tabular}

Miscelleneous drugs used are as follow, in respiratory conditions were bronchodilator, systemic steroid and nasal decongestant; CNS disorders antiepileptic and diuretics; cardiovascular disease NSAID, inotropic, antihypertensive, diuretics; genitourinary tract infections alkalizing agent and steroid and GIT disorders minerals, rehydration fluids, antispasmodic probiotic, which has been shown in Table 2. Antimicrobial prescription details of patients according to percentage has been shown in (Table 3).

\section{Table 3: Antimicrobial prescription distribution.}

\begin{tabular}{|ll|}
\hline Patients received single antimicrobial & $\mathbf{4 6}$ \\
\hline Patients received two antimicrobials & 25 \\
\hline Patients received three antimicrobials & 15 \\
\hline Patients received four antimicrobials & 2 \\
\hline Patients received five antimicrobials & 2 \\
\hline Patients did not received antimicrobials & 10 \\
\hline Total number of patients & 100 \\
\hline
\end{tabular}

The majority of the therapy was given empirically based on clinical judgement. Only $6 \%$ cases culture reports showed positive results which includes two out of three samples of blood culture and sensitivity report showed the colony of staphylococcal aureus and one of klebsiela, whereas two out of three samples of urine culture and sensitivity showed enterobacter species and one of Pseudomonas aeuroginosa.

The ADR with causality addressed as possible using Naranjo scale were noted in two cases, both of them were skin rash because of Vancomycin and Tobramycin and reactions disappeared after discontinuation of the drug. ${ }^{7}$

\section{DISCUSSION}

In present we observed the gender ratio F:M (46:54) and median age of 6 years of the participants. In our study, cehaplosporins $(43.6 \%)$ were most commonly prescribed antimicrobials followed by aminopenicillin $(21.6 \%)$ and aminoglycoside. Ceftriaxone (42;56\%), Amoxyclav 
(20.3\%) followed by metronidazole $(9.3 \%)$ and amikacin $(4.6 \%)$ were most frequently prescribed similar results in the Kathmandu valley study also reported that cephalosporins were the most commonly prescribed antibiotics followed by penicillin. Among the cephalosporin, ceftriaxone were commonly prescribed. ${ }^{2}$ A study carried out in a tertiary hospital of Bangladesh reported that ampicillin, gentamycin, amoxicillin, cloxacillin and ceftriaxone were commonly prescribed. ${ }^{8} \mathrm{~A}$ similar study carried out in eastern Nepal reported that gentamycin, ampicillin, crystalline penicillin and cefotaxim were commonly prescribed antibiotics whereas in our study ceftriaxone and amoxicillin were prescribed from same class. ${ }^{9}$ A study in Chandigarh showed that aminoglycosides (Amikacin), cephalosporins (cefotaxime), quinolones (ciprofloxacin) and cloxacillin were commonly prescribed and our study shows that ceftriaxone (cephalosporins) were prescribed. ${ }^{10}$ From rural Maharashtra it was reported that commonly prescribed antimicrobial agent was cefotaxime $(21.7 \%)$ in medicine and metronidazole in surgery $(30.6 \%)$ in wards and in our study it was ceftriaxone (cephalosporine), hence the pattern of antibiotic prescription so far in different studies exhibits overall similar trends in indoor patients were according to National treatment guidelines for antimicrobial use in infectious diseases. ${ }^{11,12}$ The most common indication of antimicrobial used in our study was lower respiratory tract infections and antimicrobials prescribed were mostly from Aminopenicillin class followed by cephalosporins and aminoglycoside. A study from Nepal done in paediatric hospital of Kathmandu valley and similar study done in east Indian teaching hospital showed Cephalosporin was the top most frequently prescribed antibiotics followed by penicillin group. ${ }^{2,13}$ A study from Gujrat showed that highest number of antimicrobials were prescribed in respiratory tract infections and majority drugs used were from beta lactam and aminoglycosides group. ${ }^{14}$

In our study we observed that $(46 \%)$ received single antimicrobial agent however which was different than study carried out in Kathmandu valley reported that (93\%) of patients was prescribed one antibiotic. ${ }^{2}$ Prescribing of two or more antimicrobial was common $(60 \%)$ in paediatric cases, where as in our study it came out to be $(25 \%)$ for two and (15\%) were prescribed three antimicrobials. $^{10} 43 \%$ of cases was prescribed antibiotics. $^{15}$

Similarly another study done, showed that $55 \%$ cases were prescribed antibiotics, which shows that antibiotics are being used in a higher spectrum. ${ }^{16}$

In our study commonly used miscellaneous drugs bronchodilator, systemic steroid and nasal decongestant and in CNS were antiepileptic and diuretics; in cardiovascular disease NSAID, inotropic, antihypertensive, diuretics; in genitourinary tract infections alkalizing agent and steroid and in GIT disorders minerals, rehydration fluids, antispasmodic probiotic were used, similar trend was observed in a north Indian teaching hospital. ${ }^{13}$

In our facility initial treatment of choice was empirical and antimicrobial treatment was guided by the results of laboratory test in (25\%) with low yield of positive culture results, as similar scenario was observed in other studies. ${ }^{2,13}$

In our study, suspected $\mathrm{ADR}_{\mathrm{s}}$ were noted in only $2 \%$ cases showing cutaneous reactions which could be managed after stoppage of treatment. Study found $2.26 \%$ cases of ADRs in pediatric patients mainly cutaneous reactions or loose stools. ${ }^{13}$ Study found that found that antibiotic was responsible for large number of $\mathrm{ADR}_{\mathrm{s}}$ such as urticaria and other rashes were common. ${ }^{17}$

This study has a few limitations like, sample size was limited, and study was only conducted in one facility, therefore the results may not be generalized, and we excluded patients from intensive care units and outpatient department.

\section{ACKNOWLEDGEMENTS}

Authors would like to grateful to resident doctors from Department of Paediatrics of MGM Hospital Kamothe, Navi Mumbai, India

Funding: No funding sources Conflict of interest: None declared

Ethical approval: The study was approved by the Institutional Ethics Committee

\section{REFERENCES}

1. World Health Organization. The Selection of essential drugs. Technical Report Series no. 615, World Health Organization Expert Commitee. WHO GENEVA: Switzerland; 1977.

2. Palikhe. Prescription pattern of antibiotics in pediatric hospital of Kathamandu valley. J of Nepal Health Research Council. 2004;2(2):31-6.

3. Sanz EJ, Bergman U, Dahlstom. Pediatric drug prescribing. Eur J of Clinical Phar.1989;(37):65-8.

4. Summers RS, Summers B. Drug prescribing in pediatrics. Amtrop Paediatr. 1986;(6):129-33.

5. Ansari KU, Singh S, Pandey RC. Evaluation of prescribing pattern of doctors for rational drug therapy. Indian J of Phar. 1998;(30):43-6.

6. How to investigate Drug use in Health Facilities. Selected drug use indicators. Geneva: World Health Organization; 1993:10.

7. Naranjo CA. A method for estimating the probability of adverse drug reactions. Clin Pharmacol Ther. 1981;30(2):239-45.

8. Akter FU, Heller D, Smith A, Rahman MM, Milly AF. Antimicrobial use in paediatric wards of teaching hospital in Bangladesh. Mymensingh Med J. 2004;13:63-6. 
9. Kafle KK. Prescribing and describing practices in PHC facilities of Tarai Dist of Nepal. J of The Institute of Med. 1996;(18):61-8.

10. Sharma D, Reeta K, Badyal DK, Garg SK, Bhargava VK. Antimicrobial prescribing pattern in an Indian tertiary hospital. Indian $\mathrm{J}$ Physiol Pharmacol. 1998;42:533-7.

11. Deshmukh V, Khadke V, Patil A, Lohar P. Study of prescribing pattern of antimicrobial agents in indoor patients of a tertiary care hospital. Int $\mathbf{J}$ Basic Clin Pharmacol. 2013;2:281-5.

12. National treatment guidelines for antimicrobial use in infectious Diseases. Published by National Centre For Disease Control, 2016; version 1.0.

13. Baidya S, Hazra A, Datta S, Das AK. A study of antimicrobial use in children admitted to pediatric medicine ward of a tertiary care hospital. Indian $\mathbf{J}$ Pharmacol. 2017;49:10-5.

14. Prajapati V, Bhatt JD. Study of prescribing pattern of antimicrobial agents in the pediatric wards at tertiary teaching care hospital, Gujarat. Indian J Pharm Sci Res. 2012;3:2348-55.

15. Kafle KK, Pradhan Y, Shrestha AD, Kar KSB. Drugs use in PHC facilities of Kathamandu. J of The Institute of Med. 1993;(14):318-26.

16. Principi N. Control of antibiotic therapy in pediatric patients. Developmental Pharmacology and Theraputics. 1981;(2):145-55.

17. Priyadharsini R, Surendiran A, Adithan C, Sreenivasan S, Sahoo FK. A study of adverse drug reactions in pediatric patients. J Pharmacol Pharmacother. 2011;2:277-80.

Cite this article as: Bamel VV, Shahani SR, Mohanty NC. Study of drug utilization and antimicrobial prescription pattern of indoor paediatrics cases in tertiary care hospital. Int J Basic Clin Pharmacol 2018;7:98-102. 